\title{
PROCEEDINGS OF THE SECOND MEETING OF THE CENTRAL SOCIETY FOR CLINICAL RESEARCH HELD IN CHICAGO, NOVEMBER 22, 1929
}

Response of the Reticulocytes in the Treatment of Two Patients with Pernicious Anemia Fed Organic Material Obtained from the Suprarenal Cortex. L. G.

Zerfas, M.D., Indianapolis, and Alfred KoeHLer, M.D., Chicago.

An organic substance obtained from the suprarenal cortex, when fed to two patients with primary anemia during relapse, in amounts of $33 \mathrm{mgm}$. three times daily, produced reticulocyte responses similar to those obtained with the feeding of liver, kidney, stomach or fractions of liver. Following the reticulocyte resporkse, the red blood cells increased in number approximately equal to the number of young cells appearing in the blood on the day of the peak.

There was little, if any, clinical improvement in the patients, and the continued feeding of the preparation did not produce a further increase in the total number of red blood cells.

Subsequently, these patients were given adequate daily amounts of liver extract no. 343 , and in each instance the clinical improvement was marked and the level of the red blood cells reached normal.

This suggests the possibility that the mechanism of producing a remission of the red blood cells in primary anemia is not entirely dependent upon the initial delivery of reticulocytes into the circulation, but is probably associated with a growth-promoting substance not present in the organic material obtained from the suprarenal cortex, but present in liver, kidney, and potent fractions of liver.

Treatment of the Anemia of Myxoedema with Liver Extract. By CyRus C. STURGIS, M.D., and RaphaEl IsaAcs, M.D., Ann Arbor, Mich.

Liver extract produced a typical pernicious anemia-like reticulocyte response in a patient with anemia (red blood cells $1,300,000$ per cubic millimeter) of myxoedema. The blood differed from that of typical pernicious anemia in the sparsity of poikilocytosis and the normal icterus index. Previous to the development of the severe anemia the basal metabolic rate was minus 30 per cent. When the anemia was most marked the rate was normal. With subsequent decrease in the anemia, the rate fell to minus 25 per cent. The increased metabolic activity of the processes associated with the anemia masked the decreased metabolism associated with the myxoedema.

.The Morphology of the Blood in Pernicious Anemia with Special Reference to the Polymorphonuclear Neutrophile. By Frank J. Heck, M.D., and Charles H. Watkins, M.D.

Very little attention has been paid in this country to the morphologic diagnosis 
of pernicious anemia. In a study of 115 cases of pernicious anemia which had had inadequate treatment or no treatment at all, only three cases failed to show an increase in the lobulation of the polymorphonuclear leukocytes, or a shift to the right according to the Cooke modification of the Arneth count. In the presence of a macrocytosis and a right shift in the polymorphonuclear leukocytes a diagnosis of pernicious anemia can be made with a high degree of accuracy. These changes are frequently present even though the blood count is normal or nearly so.

The Ante-Mortem Localization of Coronary Occlusion and Myocardial Infarction by Means of the Electrocardiogram. By A. R. BARnes, M.D., Rochester, Minn.

Combined pathologic and electrocardiographic studies in coronary occlusion and myocardial infarction showed that infarction in the posterior basal portion of the left ventricle and the adjacent interventricular septum yielded electrocardiographic changes that were characteristically different from those obtained when the infarction involved the anterior portion of the left ventricle or the apex. The electrocardiographic findings which characterize infarction in these two regions are presented. Using these electrocardiographic criteria it was possible in three cases reported to predict during life the situation of infarction and the prediction was verified at necropsy. Inasmuch as the posterior basal portion of the left ventricle and the adjacent portion of the interventricular septum usually are supplied by the right coronary artery and the remainder of the left ventricle usually is supplied by the left coronary artery, it was possible to predict which coronary artery was occluded in these three cases.

Sphygmographic Differentiation of Aortic Insufficiency Caused by Syphilis from that Caused by Rheumatic Fever. By Harold FeIl, M.D., Cleveland, Ohio.

The subclavian pulse was studied in various types of aortic insufficiency by the Wigger's optical method. In fifteen instances the etiological factor was confirmed by autopsy. The curves of aortic insufficiency caused by rheumatic infection rose abruptly but the anacrotis was usually interrupted by a notch up one-third the ascent. Maximum ejection was not reached until the end of systole showing a modified stenosis contour. The summit was interrupted by vibrations. The curves in luetic aortic regurgitation rose more abruptly and the sharp peak was more rapidly attained-the curves falling away sharply. Rheumatic curves show evidence of some stenosis while luetic curves showed little or none. These findings were confirmed by autopsy.

Electrocardiographic Changes in Anoxemia of the Heart. By William B. Kountz, M.D., and Charles M. Gruber, M.D., St. Louis Mo.

In a study of the electrocardiogram of a dog in the state of general anoxemia it was found that the complexes resembled those described in coronary occlusion. Vasoconstrictor substances by coronary constriction accentuated the picture to a most extreme degree. The " $T$ " wave passed from a large prominent type to a 
direct branching from the down-stroke of the "QRS" complex. Vasodilator substances and oxygenation of the blood caused a complete disappearance of the electrical phenomena.

A similar curve to this was obtained by Samajloff upon injury to the heart, but its effect was only recorded when the electrodes were placed directly on the heart muscle. He attributed the change to the influence of the current of injury upon the electrocardiogram.

We suggest that the high branching of the " $T$ " wave seen is a result of anoxemia of the heart muscle.

Statistical and Clinical Observations in Support of the New Interpretation of Electrocardiograms of Bundle Branch Block. By A. GarRard MACLEOD, M.D. (by Invitation), and PaUl S. BARKer, M.D., Ann Arbor, Mich.

By recording ventricular extrasystoles produced by electrical stimulation of known locations on the exposed human heart, Barker, Macleod, Alexander and Wilson (paper presented before the Association of American Physicians May 7, 1929) have shown that what has previously been considered right bundle branch block is in reality left and vice versa.

Adopting this revised terminology our studies of a large collection of electrocardiograms and case records show:

1. Concordant as well as discordant curves may characterize bundle branch block. Concordant curves are relatively more common in right bundle branch block than in left bundle branch block.

2. The relative incidence of right and left bundle branch block corresponds to the relative incidence of right and left ventricular disease, and right and left ventricular preponderance:

3. All cases with right bundle branch block had either diffuse myocardial disease or right ventricular disease, and all cases of left bundle branch block had either diffuse myocardial disease or left ventricular disease.

4. Some patients with left bundle branch block had previous curves which did not show defects in intraventricular conduction. These were either normal or showed left ventricular preponderance. The old paradox of patients with left ventricular preponderance developing right bundle branch block is thus done away with.

5. Only two of the patients with bundle branch block which came to autopsy revealed anything significant. In both cases the pathologic findings supported the revised rather than the original diagnosis.

Studies on the Circulation, Injection Method: Clinical Applications. By JoHN WALKer MOORE, M.D., and (by Invitation), W. F. Hammton, M.D., J. M. Kinsman, M.D., and R. G. SpurLing, M.D., Louisville.

For two and one-half years we have been working on a procedure which we hope will shed light upon the physiology of the circulation. By an injection method the cardiac output, circulation times and intra-thoracic blood volume can be 
determined. It is proposed to discuss certain significant variation in these quantities in health and disease.

\section{"Malignant Bypertension": A Clinical and Morphological Study. By Francis} D. MurPhy, M.D., and JoHN GriLl, M.D., Milwaukee, Wis.

This investigation is an attempt to determine whether so called malignant hypertension is a separate and distinct form of disease, as many claim, or only a severer grade of what is frequently called benign hypertension. We have tried to decide whether there are uniform changes in the vascular system distinctive of the condition as described by some investigators.

Of the seventeen patients studied, thirteen died and twelve were autopsied; two are under observation and one failed to report for further study. Clinically the features of all cases were substantially the same. The outstanding features were a continued hypertension irresponsive to usual treatment, a rapid course ending fatally within a period that varied from a few months to two years. Cardiac hypertrophy was present with very little reduction in renal function until late in the course of the disease. A decided retinopathy was always found.

Histologically, the changes in the blood vessels of all organs were studied especially, and described in full in the complete report. There was a partial or complete obliteration of the lumina of the smallest arteries and arterioles of most of the organs of the body. The lesions in the walls of the vessels consisted in a profound arteriosclerosis. There were various degrees of fatty and hyalin degeneration, connective proliferation of the intima, and hyperplastic elastic thickening of the intima. Occasionally the media was thickened due at times to hypertrophy of the muscle, and at times to fibrous tissue proliferation or fatty degeneration. Fatty degeneration and hyalinization were found in many glomeruli.

It was concluded from this study that "malignant hypertension" is not a separate and distinct disease but only a rapidly advancing form of what is usually described as "essential" or "benign" hypertension. Histologically the chief change is an advanced arteriosclerotic process involving the smallest arteries and arterioles of many organs of the body.

The Effect of Nitrites on the Gastro-Intestinal Tract. By A. J. BEAMs, M.D., Cleveland, Ohio.

Abdominal pain was controlled temporarily by nitrites when the pain was caused by spasm or hyperperistalsis of the stomach or intestine.

Fluoroscopic studies of the gastro-intestinal tract in over 200 patients, revealed a decrease of tone and cessation of peristalsis when nitrites were administered.

Experimental studies were made on animals to determine the manner and localization of the action of nitrites (1) by fluoroscopic and x-ray studies of the gastrointestinal tract, (2) direct observations on the stomach and intestines, (3) on the excised strips of intestine.

The evidence obtained by this study indicates (1) that nitrites act directly on the smooth muscle of the intestines, (2) nitrites are preferable to atropin as an anti-spasmodic of the gastro-intestinal tract. 
The Relation Between the Acidosis and Uremia in Chronic Interstitial Nephritis. By A. P. Briggs, St. Louis, Mo.

Koehler ${ }^{1}$ has shown that administration of phosphoric acid in doses of 15 to 20 grams daily to individuals with normal kidney function causes a severe acidosis within about a week. After a plasma $\mathrm{CO}_{2}$ of about 25 had been maintained for two or three days the following symptoms began to appear: headache, drowsiness, stupor, loss of appetite, nausea, and vomiting. These are the symptoms found by Feinblatt ${ }^{2}$ in a study of a large group of nephritics to be especially characteristic of uremia. In cases in which uremia does not appear until high retention levels of urea, phosphoric acid, and the other excretory substances have been reached, it is conceivable that the symptoms may be secondary to the acidosis.

In chronic interstitial nephritis uremia may appear with a relatively low N.P.N. and only a mild acidosis. It seems that the arteriosclerosis present in this type of nephritis might be an important factor working with the acidosis to cause uremia from the following considerations. Koehler points out that the symptoms are those of tissue asphyxia and that a severe prolonged acidosis tends to cause tissue asphyxia. The arteriosclerosis by cutting down blood flow also tends to cause tissue asphyxia. The fact that the symptoms are chiefly referable to the brain suggests that the centers in the brain are sensitive to slight fluctuations in blood flow or $\mathrm{pH}$.

From the foregoing it would seem in chronic interstitial nephritis that administration of phosphoric acid in doses easily tolerated by normal individuals might cause the appearance of uremia, and that administration of certain alkaline salts in the presence of uremia might to a certain extent alleviate the symptoms.

Studies designed to test these points have shown that administration of phosphoric acid in doses of 6 grams daily in cases with N.P.N. over 60 causes the appearance of headache, drowsiness, and nausea within three or four days. The administration of this relatively small dose of phosphoric acid to these patients causes a distinct drop in the level of the plasma $\mathrm{CO}_{2}$ and a rise in the inorganic phosphorous, which is not surprising on account of their poor kidney function. However they appear to develop the symptoms more promptly than a normal individual at a given level of acidosis, perhaps because they are not able to compensate so well by an increase in blood flow on account of their blood vessel disease. Attempts at alkaline therapy have not been very fruitful. On account of nausea and vomiting, if not coma, medication must be parenteral. Transient improvement frequently reported from sodium bicarbonate seems to be in harmony with views expressed above. But the sodium salts formed in the tissues are not promptly eliminated and prolonged therapy causes edema, sodium poisoning and even alkalosis. Calcium and strontium salts are promptly eliminated in the feces but have to be given cautiously and in small doses by parenteral routes.

${ }^{1}$ Koehler A. E., J. Biol. Chem., 1927, lxxii, 99.

${ }^{2}$ Feinblatt H. M., Boston Med. and Surg. J., 1923, clxxxix, 399. 
The Use of a Single Solution in the Treatment of Acidosis, Alkalosis and Dehydration. By Alexis F. Hartmann, M.D., St. Louis, Mo.

The most important clinical forms of dehydration result from loss of electrolyte from the body by way of the gastro-intestinal tract (vomiting, lavage, obstruction, fistula, diarrhea, etc.) or urine (polyuria, renal insufficiency). Marked dehydration may be associated with either very marked acidosis or alkalosis, or the changes may be so balanced that a normal $\frac{\mathrm{BHCO}_{3}}{\mathrm{H}_{2} \mathrm{CO}_{3}}$ ratio is maintained.

If the following solution is administered parenterally

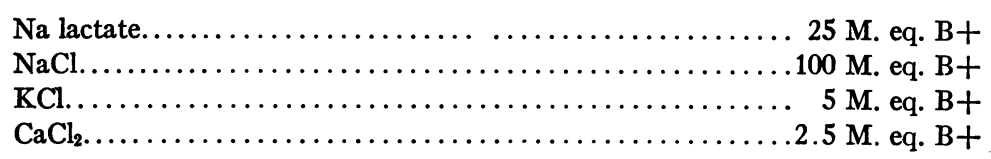

relief of dehydration is coincident with disappearance of either acidosis or alkalosis. Résumé of experimental and clinical evidence.

The Use of a Balanced Diet, Acid and Acid-Producing Salts in the Treatment of Nephritic Edema. Preliminary Report. By F. H. LASHMET, M.D. (by Invitation) and L. H. NewBurgh, M.D., Ann Arbor, Mich.

The mechanism of the reduction of edema of nephritic origin by the use of acid and acid-producing salts is discussed. A diet which improves the diuretic action of these substances and which is balanced in inorganic salt content is described. Especial emphasis is placed upon the necessity of this balanced diet with regard to the alkaline and acid ash in promoting diuresis by these salts. Several cases in which this combined procedure was employed are reported.

Sodium Ricinoleate in the Treatment of Intestinal Allergy of Bacterial Origin. By Stanley E. Dorst, M.D., and Roger S. Morris, M.D., Cincinnati, Ohio.

The paper is introduced by a brief abstract of our sensitization studies establishing the reasons for employing entodermal tests as an index of specific bacterial allergy. We will review a series of experiments demonstrating the toxicity of various members of the enteric flora and attempt to show that the so-called "normal flora" is not innocuous but is, instead, frequently the cause of marked clinical symptoms.

We will then show the effects of sodium ricinoleate in detoxifying these organisms and demonstrate the antigenic action of strains so detoxified.

Non-specific Factors Influencing the Tuberculin Skin Reaction. By A. GraEmE Mitchell, M.D., Cincinnatti, Ohio.

A review of the literature dealing with the effect of non-specific factors on the tuberculin skin reaction shows that comparatively little work has been done. While many statements are made they are frequently not substantiated by suff- 
cient evidence. It is, however, stated that the tuberculin reaction may become negative in the acute stage of measles, German measles, chickenpox and influenza -becoming positive again during convalescence. Concerning the effect of pertussis there is a difference of opinion. Local factors such as anemia, water content of the skin, light and the like are said to influence the intensity of the reaction. It is stated that the injection of foreign protein causes an increased skin response.

Our own studies are summarized in the following table.

Tuberculin test during acute and convalescent stages of diseases

\begin{tabular}{|c|c|c|c|c|c|}
\hline \multirow{2}{*}{ Disease } & \multirow{2}{*}{$\begin{array}{l}\text { Number } \\
\text { of cases }\end{array}$} & \multicolumn{2}{|c|}{ Febrile stage } & \multicolumn{2}{|c|}{ Convalescent stage } \\
\hline & & $\begin{array}{l}\text { Number } \\
\text { positive }\end{array}$ & $\begin{array}{l}\text { Per cent } \\
\text { positive }\end{array}$ & $\begin{array}{l}\text { Number } \\
\text { positive }\end{array}$ & $\begin{array}{l}\text { Per cent } \\
\text { positive }\end{array}$ \\
\hline Measles.................. & 200 & 7 & 3.5 & 30 & 15.0 \\
\hline Diphtheria.............. & 105 & 13 & 12.0 & 21 & 20.0 \\
\hline Poliomyelitis........ & 38 & 3 & 8.0 & 4 & 10.5 \\
\hline Scarlet fever.......... & 435 & 17 & 4.0 & 84 & 19.0 \\
\hline Varicella......... & 52 & 4 & 8.0 & 12 & 23.0 \\
\hline Pertussis.............. & 72 & 3 & 4.0 & 3 & 4.0 \\
\hline Miscellaneous............ & 32 & 2 & 6.0 & 5 & 16.0 \\
\hline Total. . & 934 & 49 & 5.0 & 159 & 17.0 \\
\hline
\end{tabular}

In these studies the same type and amount of tuberculin was always employed in the same patient during the febrile and during the convalescent stage.

It also developed in the course of the study that the degree of local reaction during the acute stage was often less than the same amount of tuberculin would cause during convalescence; that a positive reaction could sometimes be secured during the acute stage by employing a larger dose of tuberculin than was necessary later; that in some cases the appearance of the skin reaction was delayed for three or four days or longer during febrile stages of disease. Tables were given which showed that the Dick and Schick tests were somewhat more frequently positive during the convalescent stage of certain diseases than during the febrile stage, but the difference was not as marked as in the case of the tuberculin reaction.

It was concluded that a positive skin tuberculin reaction is approximately two to four times as frequent during the convalescent afebrile stage as during the acute stage of such diseases as measles, diphtheria, scarlet fever, varicella, poliomyelitis, pneumonia, influenza, gastro-enteritis and the like. There is some evidence that larger doses of tuberculin may cause a positive reaction to occur during the febrile stage of these diseases. Readings of tuberculin reactions during acute diseases should be continued for several days as the skin response may be delayed longer than forty-eight hours. The effect of temperature, rather than of any specific disease, seemed to be the important factor. 
Unusual Reactions to Protein Therapy. By PhIlIP S. Hench, M.D., Rochester, Minn.

Certain profound clinical and physiological alterations generally follow the intravenous administration of typhoid vaccine used as a form of protein therapy. The reaction induced however, is usually entirely harmless and unattended with danger or untoward clinical manifestations aside from the well-known phenomena of chill, fever, malaise, and mild gastro-intestinal upsets. Occasionally however, unusual reactions occur which demand special recognition and treatment. Fifteen examples of unusual "focal" and other reactions are discussed: acute or subacute appendicitis, cholecystitis, enteritis, iritis, glaucoma, pleurisy, pericarditis, adenitis, extensive vascular thrombosis and renal insufficiency. Fatal terminations in three instances are detailed. Some of the possible causal factors are outlined; the management of such unusual reactions is discussed and conclusions given concerning contraindications for such therapy.

Streptothrix Infection of the Lung with Metastases to all Organs including the Skeleton. By J. J. Singer, M.D., and (by Invitation), Harry C. Ballon, M.D., and Grace Mehrten, M.S., St. Louis, Mo.

An unusual case of what is considered to be a primary streptothrix infection of the lung in a white male, 32 years of age, who developed "streptothrix metastases" in practically every organ and structure in the body including the skeleton, is presented.

The course of the patient's disease during his three stays in the hospital separates itself out into three clinical stages, each of which strangely enough coincides with one or other of the three admissions. The first of these was on October 8 , 1928, when a diagnosis of chronic interstitial nephritis and hypertension was made. The patient's complaints on this occasion were oedema of both lower and upper extremities, polyuria, fatigue and pain in the right buttock. Physical examination of the lungs was negative, although a roentgen ray plate of the chest taken at the time suggested a diagnosis of pulmonary tuberculosis. Discharged on October 15, 1928, the patient was readmitted on December 1, 1928. He was then found to be suffering from purpura which had just begun to make its appearance at the end of his former admission, and which was considered to be an allergy to wheat. At first a wheat free diet caused the disappearance of the oedema and purpura and its addition to the diet provoked their reappearance, but later this therapeutic test called forth no response. The patient also complained of back pain. A compression fracture of the seventh thoracic vertebrae (? Kummel's disease) was then discovered. A back brace which was supplied for the spine condition brought some relief. Following his discharge from Barnes Hospital on December 14, 1928, the patient visited the Johns Hopkins Hospital. Numerous fractures of the pelvic bones and an ulcer on the left chin, traumatic in origin which refused to heal, were then discovered. He was cystoscoped but the roentgen ray pictures which were taken following the injection of bromide revealed normal renal pelves on both sides. 
Three months previous to his final admission to Barnes Hospital which was on May 5, 1929, the patient developed a cough, brownish expectoration and pain referable to his right chest. Physical signs and roentgen ray suggested a lesion in the right chest. A small swelling just over the tenth rib, right, posteriorly, was then discovered. It was aspirated and pus obtained. Streptothrix in pure culture was recovered from this pus. Animals were innoculated and typical lesions were produced. Stained sections of these lesions showed streptothrix. Several abscesses of the chest wall which did not appear to communicate with the pleural cavity but which lay over spontaneously fractured ribs were drained. The patient's spine collapsed during the period between his second and third admission. His height, originally over five feet six inches, was reduced to considerably less than five feet.

Streptothrix was recovered from several abscesses, and from the sputum. Blood and urine cultures were negative. The patient made no response to large doses of potassium iodide and expired on June 14, 1929. Complete necropsy revealed a generally disseminated streptothrix infection. The lungs and various organs showed the characteristic lesions. Many of these represented rather the earliest stage of the lesion, namely, greyish tubercle like nodules. The fractures of the pelvic bones were healed. Streptothrix could be recovered from the various organs including the bone marrow.

Undulant Fever: A Clinicopathological Study of Eighty Cases Occurring in and about Dayton, Ohio. By Walter M. Simpson, M.D., Dayton, Ohio.

Medical literature of the past three years has brought an overwhelming abundance of evidence as to the widespread distribution of undulant fever in this country. Data have now been collected on over 1,000 cases, the great majority of which have been recognized during the past three years. It has become increasingly apparent that the disease is invariably found to be widely prevalent when an earnest effort is made to learn its incidence in a given locality. Eighty confirmed cases of undulant fever, occurring in and about Dayton, Ohio, have been studied by the writer during the past two years.

Most of the published work on undulant fever has dealt with laboratory studies. The clinical features of the disease have not been emphasized. It has been repeatedly stated that the clinical diagnosis of undulant fever is made with great difficulty. In our experience undulant fever has presented a sufficiently well-defined clinical picture to lead to an initial clinical diagnosis of undulant fever in over one-third of our cases. The purpose of this paper will be to emphasize the clinical manifestations of the disease.

In none of the Dayton cases was there any evidence of direct caprine or porcine origin. The relationship between the ingestion of raw milk containing Brucella abortus and the development of undulant fever in human beings was established in several of our cases. 
Studies on Human Skin Temperature. II. Some Observations on Changes in the Skin Temperature in Myxoedema and Hyperthyroidism. By CharLes L. Brown, M. D., and (by Invitation), J. Feigenbaum, M.D., Ann Arbor, Mich. The study reported here represents observations on a special group of cases, namely those in which the thyroid gland is particularly at fault. Cases of myxoedema and hyperthyroidism were studied. The latter group included both exophthalmic goitre anid toxic adenomatous goitre. Normal individuals were used as controls.

The temperature determinations were made by the thermo-electric method using thermal junctions and galvonometer (as described by Dr. F. G. Benedict).

Forty-three points on the surface of the body were selected and determinations of the skin temperature were made at these points on successive days. In general, several determinations were made before treatment was started. Then the influence of treatment was observed, namely that of desiccated thyroid in the cases of myxoedema and that of Lugol's solution and thyroidectomy in the cases of hyperthyroidism. In addition, an opportunity was afforded to correlate the influence on skin temperature of certain environmental factors, such as room temperature and humidity.

The Production of Hyperplasia of the Thyroid Gland by Chemical Means with Special Reference to Purine Bases, Their Derivatives and Amino Acids. By WARREN H. Cole, M.D., and (by Invitation), Nathan A. WoMaCK, M.D., and CALEB. S. Stone, JR., M.D., St. Louis, Mo.

In attempting to explain the effect of certain infections and toxemias on the thyroid (hyperplasia, desquamation, loss of colloid and iodine content) which we and others have observed, we encountered certain noteworthy facts. The methylpurines, caffeine and theophylline will produce a hyperplasia of the thyroid cells on certain occasions, which is almost indistinguishable from the hyperplasia seen in the toxic goitres of human beings. We feel that since these drugs are so similar to the products of protein catabolism (specially those of nucleoproteins) the effect may be dependent upon toxic by-products formed during protein destruction. The thyroid of an animal which had received an amino acid, tyrosine, in doses insuffcient to produce toxic effect showed a marked degree of desquamation and loss of colloid. It must be admitted and emphasized that the results obtained from the administration of most of the drugs used in this series are subject to correction since many of the drugs were used on only one or two occasions. We do feel, however, that the production of a marked degree of hyperplasia following administration of certain drugs is significant, even though it does not occur in every instance. As a whole, inorganic drugs apparently do not produce many changes in the thyroid of animals even after administration of fatal doses. The results contained in this report are preliminary. Further investigation is being done on the effects of drugs, especially by-products of protein destruction, upon the thyroid gland of animals. 
A Study of Bile Salts in Normal and Jaundiced Urines. By G. O. Broun, M.D., and A. P. Briggs, M.D., St. Louis, Mo.

To $20 \mathrm{cc}$. of urine is added $1 \mathrm{cc}$. of a concentrated solution of ferric sulfate or lead acetate. The mixture is centrifuged and the supernatant fluid transferred to another tube. After alkalinization with sodium hydroxide the resultant precipitate is removed by centrifugation. The supernatant fluid after transfer to a separatory funnel is made acid with sulfuric acid. Twenty cubic centimeters of butyl alcohol are added and the mixture shaken vigorously. After allowing the layers to separate the aqueous layer is discarded. The butyl alcohol layer is freed of pigment by shaking successively with very dilute sulfuric acid, 10 per cent sodium hydroxide $\mathrm{N} / 10$ sodium hydroxide, and finally very dilute sulfuric acid. It is then transferred to a small beaker and a quantity of magnesium carbonate sufficient to insure neutralization is added. It is evaporated to dryness on a glycerin bath without removal of the excess magnesium carbonate. The dry residue is twice extracted with $5 \mathrm{cc}$. of boiling water. The extracts are filtered through a small filter paper. A modified Pettenkoffer reaction similar to that described by Gregory and Pascoe is then performed for the detection of the bile salts and their quantitation. Fairly pure blue colors are usually obtained but sometimes a slight brownish discoloration mars the color.

All normal urines so far tested have given no evidence of the presence of bile salts. Some jaundiced urines have definitely given positive reactions. Other jaundiced urines from proved cases of hepatic pathology have given negative tests.

The Significance of Lead in the Urine in Patients with Peripheral Vascular Disease. By Bayard T. Horton, M.D., and Myron H. Powelson, M.D., and A. E. Osterburg, Ph.D., (by Invitation), Rochester, Minn.

Lead is an abnormal constituent of urine. Fifty cases of peripheral vascular disease were studied, 85 per cent of which showed lead in the urine. In this number were included thrombo-angiitis obliterans, Raynaud's disease, arteriosclerosis, scleroderma and essential hypertension. 'The patients' symptoms were not suggestive of lead poisoning, and there was no history of contact with lead except in a few cases. An adequate series of normal subjects have been studied in a similar manner.

Lead has long been known as one of the causes of arteriosclerosis, but so far as we are aware, it has never been associated with the other group of vascular diseases. In the average case from 0.5 to $1 \mathrm{mgm}$. of lead was excreted in the urine in twentyfour hours. The large vessels in the extremities, as well as the small ones, were involved in the occlusive and spastic processes. Spasm was an outstanding feature, especially in the large vessels, and graphic records were obtained to illustrate this point. The significance of lead as a vascular poison is considered.

Spectrophotometric Absorption Curves of Rabbit Blood after Immunization with Lens Solution. By F. Lowell DunN, M.D., Omaha, Neb.

The absorption curves were determined over the region of 220 to 7000 A.U. for 
normal rabbit blood plasma and the globulin fraction. The rabbits were immunized with alpha crystalline lens protein and the complement fixation and precipitin tests were run. Curves after immunization showed no consistent deviations from the normal. Absorption curves of tetanus and diphtheria antitoxin showed no significant differences.

Relation of Age in the Effect of Atropine. By C. F. G. BRown, M. D. (by invitation) and STUART P. Cromer, M.D. (by invitation), Chicago.

Effects of atropine sulphate on normal people of different ages were investigated. Preliminary examination of results showed that the difference in the different decades was quite noticeable.

An increased atropine effect with increased ages was noticed with salivary secretion. Indications are that pulse pressures are constant in decades up to the sixth, when they tend to lower and vary considerably. Heart rates show a definite age correlation. Oral temperatures were lowered in general in all decades after atropine. Skin temperatures showed a tendency to increase for a short time after atropine and considerable individual variation was shown. Attempts will be made to correlate results in relation to constitutional tendencies.

Observations on the Surface Capillaries after Excision of The Vasomotor Nerves. By George E. Brown, M.D., Rochester, Minn.

A group of cases of Raynaud's disease, thrombo-angiitis obliterans, and scleroderma affecting the upper extremities have been studied. Quantitative studies of the capillaries in the nail-fold areas have been studied before and after cervicodorsal sympathetic ganglionectomy. There was a definite narrowing of the visible capillary loops in the cases of Raynaud's disease and scleroderma after operation. The number of open capillaries increased for each unit area of skin. There was acceleration of the capillary flow and arteriolar spasm was greatly diminished. These observations add some proof to the conception that in certain conditions in which there is capillary stasis the dilatation of the capillaries is due to the accumulation of some vasodilating substances. Following ganglionectomy and improvement in the capillary circulation and disappearance of stasis removal of the dilating substances would follow; the changes observed in the size and caliber of the capillary loops could only be explained on this basis.

The Effect of Parotid Duct Ligation on Carbohydrate Tolerance in Dogs. By Leo M. Zimmerman, M.D. (by invitation), Chicago, Ill. (Introduced by William Thalhimer, M.D.)

The resemblance between the parotid glands and the pancreas on embryological, morphological and functional grounds has often been pointed out, and their common involvement in certain pathological conditions has been noted. From time to time evidence has been advanced that the parotids further resemble the pancreas in possessing a similar or supplementary endocrine action on the carbohydrate metabolism, and recently, the parotid ducts have been ligated in small 
groups of patients in several clinics as treatment for diabetes mellitus. In order to study the effect of ligation of the parotid ducts on the carbohydrate metabolism sugar tolerance determinations have been made before, and at varying intervals after the duct ligation in a series of normal dogs. In all of the animals studied, there was a more rapid removal of the excessive glucose from the blood after the ducts were ligated, and this action persisted, usually for the entire period of observation, which never, however, exceeded three months. Further studies are in progress to determine the source and nature of this action.

Results of Sympathetic Ganglionectomy and Trunk Resection in Chronic Arthritis.

By Leonard G. Rowntree, M.D., and (by invitation), Alfred W. Adson, M D., Rochester, Minn.

In The Mayo Clinic, sympathetic ganglionectomy and trunk resection has been performed seven times in six cases of chronic arthritis. The first operation, bilateral lumbar sympathetic ganglionectomy, was performed in June, 1926, in a case of arthritis deformans in a woman, aged thirty-four years, who had suffered from periarticular arthritis for six years. Complete cure of the arthritis of the lower extremities resulted. Cervicothoracic sympathetic ganglionectomy was then performed and this resulted in remarkable improvement in the upper extremities. Before the first operation this patient was practically bedridden and appeared to be crippled for life. There now is no evidence of chronic arthritis except that the left wrist and right shoulder are slightly affected.

The second patient was a Greek girl, aged sixteen years, who had had arthritis for two years; the last ten months had been spent in bed. She was dismisesd two months after operation, able to walk a distance of 100 yards without discomfort; all arthritic pain had disappeared. Four months later, she stated in a letter that she could walk two squares and could dance a little.

The third case was of subacute arthritis in a woman aged forty-four years. There was marked swelling and deformity of the hands. Two months after operation the appearance and function of the hands had improved remarkably.

The fourth patient was a young man, aged twenty-six years, who had been ill for ten years, and had been subjected to every known form of treatment, including arthrodesis in one knee. There was marked swelling of the knees, and extreme pain in the hip joints with marked bony changes. Six weeks after operation he could walk a distance of 50 feet.

The fifth patient, a woman, aged twenty-three years, had had arthritis for eleven years and had been bedridden for, three and a half years. The bony changes were marked. After operation, pain persisted for a considerable period, then subsided gradually, until now, after two and a half months, she is walking a distance of $\mathbf{5 0}$ to 100 feet with the help of a walker

The sixth case is that of a woman, aged thirty-five years, who had suffered from arthritis for a period of ten and a half years. Before operation she could walk a short distance but only with great pain. Within six weeks after operation, she 
walked considerably more and with much less pain. She had walked upstairs for the first time in eight years.

The morphologic and trophic changes are just as striking as the loss of pain and improvement in function. The results of these operations appear almost miraculous and would seem to be due to improvement in the circulation of the parts affected. 